\title{
AIAA 2002-0266 Flow Control Device Evaluation for an Internal Flow with an Adverse Pressure Gradient
}

L. Jenkins, S. Althoff Gorton, and S. Anders

NASA Langley Research Center

Hampton, VA

\section{$40^{\text {th }}$ AIAA Aerospace Sciences Meeting \& Exhibit 14-17 January 2002/Reno, NV}

For permission to copy or to republish, contact the copyright owner named on the first page.

For AIAA-held copyright, write to AIAA Permissions Department, 1801 A lexander Bell Drive, Suite 500, Reston, VA, 20191-4344. 
AIAA-2002-0266

\title{
FLOW CONTROL DEVICE EVALUATION FOR AN INTERNAL FLOW WITH AN ADVERSE PRESSURE GRADIENT
}

\author{
Luther N. Jenkins*, Susan Althoff Gorton ${ }^{\dagger}$, and Scott G. Anders ${ }^{\ddagger}$ \\ NASA Langley Research Center \\ Hampton, Virginia
}

\begin{abstract}
The effectiveness of several active and passive devices to control flow in an adverse pressure gradient with secondary flows present was evaluated in the 15 inch Low Speed Tunnel at NASA Langley Research Center. In this study. passive micro vortex generators. micro bumps. and piezoelectric synthetic jets were evaluated for their flow control characteristics using surface static pressures. flow visualization. and 3D Stereo Digital Particle Image Velocimetry. Data also were acquired for synthetic jet actuators in a zero flow environment. It was found that the micro vortex generator is very effective in controlling the flow environment for an adverse pressure gradient. even in the presence of secondary vortical flow. The mechanism by which the control is effected is a re-energization of the boundary layer through flow mixing. The piezoelectric synthetic jet actuators must have sufficient velocity output to produce strong longitudinal vortices if they are to be effective for flow control. The output of these devices in a laboratory or zero flow environment will be different than the output in a flow environment. In this investigation, the output was higher in the flow environment, but the stroke cycle in the flow did not indicate a positive inflow into the synthetic jet.
\end{abstract}

\section{Introduction}

The effect of aviation on the environment and in particular global warming has recently become a focus of study'. In response to environmental concerns and to foster revolutionary propulsion technologies. NASA launched the Ultra Efficient Engine Technology (UEET) program in late $1999^{2}$. This program has several elements. one of which is to explore the feasibility of the Blended-Wing-Body (BWB) concept as an efficient alternative to conventional transport configurations. The BWB concept has been considered in various forms for several years ${ }^{3-5}$. Studies have shown that in order to make the largest impact on the vehicle performance. the engines and inlets should be placed near the surface on the

*Research Engineer. Flow Physics and Control Branch

t Research Engineer. Flow Physics and Control Branch. Member AIAA

${ }^{\text {t}}$ Research Engineer. Flow Physics and Control Branch

Copyriuht (c) 2002 by the American Institute of Aeronautics and Copyright (c) 2002 by the American Institute of Aeronatics ander Title 17 US Code. The U.S Government has a royalty-free license to exercise all rights under the copyright claimed herein for government purposes. All other rights reserved by the copyright owner aft section of the vehicle. This configuration of the BWB is shown in Ref. 6 and pictured in Figure 1.

When the engines are positioned near the surface. the BWB engine inlet must be an S-duct inlet with the capability to ingest the large boundary layer that will build up over the aircraft body. The inlet must perform this task without producing a significant engine performance penalty in terms of distortion or pressure recovery. Since the boundary layer on the BWB is expected to be on the order of $30 \%$ of the inlet height. this presents a challenging task for inlet design.

The requirements for inlet performance under the severe conditions of an adverse pressure gradient from the S-duct and a very large onset boundary layer flow have led to the consideration of active flow control devices in the inlet to control the flow. As reported in References 7-25. much research is already underway to identify and develop active flow control devices and technologies and this represents only a sampling of the available material on the subject. There have also been investigations showing the successful use of passive and active flow control technologies applied to inlets. Reference 6 discusses work using passive devices for an Sduct with boundary layer ingestion (BLI). and References 18 20 discuss both passive (microvanes) and active (microjet) concepts applied to aggressive serpentine inlets.

The purpose of the present investigation was to lay the groundwork for a future study of active flow control applied to a duct representative of a BWB with BLI. In the present study, the effectiveness of several active and passive devices to control flow in an adverse pressure gradient with secondary flows present was evaluated by examining pressure recovery. flow topology, and flow-field velocity and vorticity characteristics. These data were obtained for passive micro vortex generators. micro bumps. and synthetic jets using surface static pressures. flow visualization. and 3D Stereo Digital Particle Image Velocimetry.

\section{Experimental Apparatus and Methods}

Facility and Model

The experiment was conducted in the NASA Langley 15 -Inch Low Speed Tunnel. This tunnel is a closed return. atmospheric facility used primarily for fundamental flow 
incidence of the cameras to the light sheet. all three components of velocity were measured in each PIV measurement plane through stereoscopic vector reconstruction. The light sheet was produced by a pulsed. frequency-doubled. $300 \mathrm{~mJ} \mathrm{Nd:YAG} \mathrm{laser} \mathrm{operating} \mathrm{at} 10$ $\mathrm{Hz}$. The laser could also be triggered phase-locked to the synthetic jet input signal. In this mode, the laser would fire on multiples of the synthetic jet cycle, as the laser physically could not fire at a faster rate than $10 \mathrm{~Hz}$.

At each measurement location, the PIV field of view was approximately 4 inches wide by 3 inches tall, centered along the centerline of the tunnel. The measurement location was carefully aligned with the model system, and the cameras were calibrated with an in-situ target for each location. The tunnel was seeded with atomized mineral oil injected into the flow in the tunnel settling chamber. and the particle size was approximately 5-10 microns. For all conditions. at least thirty samples of PIV data were obtained over a 3 to 6 second period and averaged. The low $\mathrm{rms}$ of the mean data indicated that this was enough data to capture the relevant flow features for this investigation. The algorithm used to process the images acquired in this investigation is described in Reference 30. Estimating the accuracy of the stereo PIV measurements is itself a matter of instrumentation research at this time: the best estimate the authors can provide for the accuracy of the PIV velocity measurements is included in Table $I$.

\section{Test Conditions}

The main test condition was established by setting the tunnel velocity to $100 \mathrm{ft} / \mathrm{sec}$. This corresponded to a local velocity of $140 \mathrm{ft} / \mathrm{sec}$ at station 57 due to the acceleration of the flow above the splitter plate. Station 57 was the farthest aft static surface pressure port location on the flat part of the splitter plate. For this reason. Station 57 conditions are used to define the onset flow to the adverse pressure gradient ramp. The boundary layer was measured at station 57 and found to have a thickness. $\delta$. of approximately 0.87 inches. The boundary profile was converted to wall coordinates and compared with Spalding s Law. Based on the agreement between the two profiles, the boundary layer was determined to be turbulent.

\section{Discussion of Results}

Data were obtained for many different configurations and test conditions during this investigation. In this paper, the basic flow over the ramp will be presented to define the baseline flow environment with pressures. flow visualization. and flow tield velocity measurements. Comparisons among the different flow control devices will then be presented with respect to the baseline to emphasize the effect of the devices on the flow environment. In the final section. details of several attempts to optimize the synthetic jet output will be given. and the jet performance in a no-flow environment will be presented.
Baseline flow field

The baseline configuration flow visualization topology is shown in Figure 7 for the freestream velocity of $140 \mathrm{ft} / \mathrm{sec}$. Although flow along the splitter plate in the tunnel is twodimensional for the most part. two large spiral nodes reveal the formation of vortical structures. This occurs when the sidewall boundary layer reacts to the adverse pressure gradient near station 61.75 on the ramp. The vortical structures are similar to what might be expected from secondary flow and vortex liftoff in a duct. so no attempt was made to control the vortices for this investigation. Rather, it was thought that the challenges of the strong vortical flow field would provide a better indication of how the flow control devices would work in a realistic inlet configuration. It should be noted that the vortices are highly unsteady and appear to have a trajectory that departs from the surface of the ramp and extends downstream in the tunnel. In addition to the vortical structures. Figure 7 also highlights other significant flow features such as a separation node. an attachment node, and evidence of reverse flow in the center of the ramp.

Figure 8 presents the centerline and spanwise surface static pressure distributions for the ramp. In Figure $8 \mathrm{a}$. the repeatability of the bascline pressure profile over a time period of two months and after two major model removals is also shown.

The centerline pressure distribution indicates separation occurring near station 64 but does not show the dramatic flow features that the flow visualization revealed. In fact. the spanwise pressure distribution in Fig $8 \mathrm{~h}$ indicates a fairly uniform and symmetric pressure pattern. In the absence of the other information from the flow visualization and PIV. this type of pressure distribution could easily be interpreted to be representative of uniform. two-dimensional flow.

Figure 9 shows velocities measured using PIV along the centerline of the ramp geometry at four longitudinal stations. Each frame consists of at least thirty samples of data acquired at the laser internal trigger frequency of 10 $\mathrm{Hz}$. The contours clearly indicate a thin region of reverse flow in the center of the ramp at station 68.00. The velocity measured very close to the surface on the centerline is plotted in Figure 10 and shows very slow moving and even reversed llow at these locations.

\section{Flow Control Devices}

As described earlier, several flow control devices were applied to the ramp in order to assess their relative ability to control the flow. Figure 11 shows the flow visualization obtained along the ramp for the MVG's and the synthetic jets. There was no flow visualization obtained for the micro bumps. Note how the MVG's create a series of strong vortices. as indicated by the dark separation lines. which reduce the influence of the sidewall vortices and allow the flow in the center of the ramp to remain attached. 
variations were attempted to increase the performance of the jets. Although the synthetic jets had been optimized on the bench top. it was thought that the optimum for flow control might not correspond to the optimum for synthetic jet operation. Additionally, it was possible that the jets were not operating in the same manner in a flow environment as they did on the bench. In order to sort out these issues, some limited parametric variations were evaluated and are discussed below.

Hole Size - Originally the synthetic jet output holes were 0.040 in. in diameter. Because the vortex generation was not strong enough, the hole diameter was increased to 0.094 in. in order to increase the mass flow through the holes. This was the largest size hole possible for the current geometry. Figure 17 shows that there was little effect on the pressure recovery due to increasing the hole size, although it was noted during the testing that the mass flow had increased substantially.

Backpressure - It was hypothesized that perhaps the reason the jet output was lower than expected was that the synthetic jet could not adequately pull in air mass during the instroke cycle in the presence of the onset flow and its pressure field. With no air ingested during the instroke, there would be little air available to pump out on the outstroke. In order to ensure that the actuator had mass available to pump out. the actuators were modified by installing small air pressure feed lines directly to the jets. A high-resolution regulator controlled the air in the lines. and various backpressures were applied to the configuration.

Figure 18 shows the pressure distribution for the zero backpressure case and two cases with backpressure applied at $60 \mathrm{psi}$ and $80 \mathrm{psi}$. Analysis of PIV velocity data for the zero backpressure case and the $60 \mathrm{psi}$ backpressure case also showed that backpressure has a minimal effect for the $140 \mathrm{ft} / \mathrm{sec}$ case with the actuators operating at $700 \mathrm{~Hz}$. There was some slight effect of backpressure when the tunnel speed was lowered to $45 \mathrm{ft} / \mathrm{sec}$ and the actuators were run at $300 \mathrm{~Hz}$. Steady blowing through the backpressure tubes without the synthetic jets operating also had no effect. These data lead to the conclusion that lack of air mass was not the primary reason for the low output of the synthetic jets in the onset flow.

Frequency - With the freestream velocity at $140 \mathrm{ft} / \mathrm{sec}$. the operating frequency of the jets was swept through a range from $200-1000 \mathrm{~Hz}$ with no noticeable effect on the pressure recovery along the ramp.

Amplitude - The amplitude of the synthetic jet input signal was swept through a range of 40-92 VAC at a freestream velocity of $140 \mathrm{ft} / \mathrm{sec}$ with no significant effect on the pressure recovery data.

Freestream lelocity - The freestream velocity was changed in a range from $45 \mathrm{ft} / \mathrm{sec}$ to $140 \mathrm{ft} / \mathrm{sec}$ with the actuators operating at $700 \mathrm{~Hz}$ and an input amplitude of 92 VAC, At the lowest velocity, $45 \mathrm{ft} / \mathrm{sec}$. the actuators appeared to improve in performance. Figure 19 shows vectors for the phase-locked output of the jets for a tunnel velocity of $140 \mathrm{ft} / \mathrm{sec}$ and $45 \mathrm{ft} / \mathrm{sec}$. In both cases. the freestream vertical velocity hias has been removed to show the operation of the jet. The vectors indicate that the jet output has essentially doubled for the higher speed condition. and the ratio of the maximum jet output to the freestream has increased from $14 \%$ in the $140 \mathrm{ft} / \mathrm{sec}$ case to $18 \%$ in the $45 \mathrm{fu} / \mathrm{sec}$ case.

No Flow Operation of the Jets - The question of whether the jets were operating as efficiently in the flow environment as they did in a laboratory environment could only be answered by measuring the output of the jets in situ with no onset flow.

Figure 20 presents the zero flow operation for the synthetic jets at $700 \mathrm{~Hz}$ and $300 \mathrm{~Hz}$ with $92 \mathrm{VAC}$ and zero backpressure. Note that the output magnitude of the jets is far less than Ref. 21 reports and also less than the output shown under the onset flow conditions in Figure 19. However. in Figure 20 there is a clear inflow and outflow stroke of the actuator that is not apparent with the flow on (Figure 19). Also the flow generated by the jets penetrates further away from the jet in the no flow condition.

The difference between the jet output in zero flow for this contiguration and that of Ref. 21 may be due to the small plenum in this configuration that increases the distance between the jet outpul slot and the surface of the ramp. However, such plenums may be necessary for realistic applications and the performance of the actuator must be improved to account for this. The results of the no onset flow measurements also show that the actuator output in a zero flow environment is lower than what is achieved in an onset flow setting. but the onset flow condition affects the penetration of the velocity into the flow as well as the phasing and stroke cycle of the actuator. These factors must be considered when the requirements for flow control actuators are determined.

\section{Conclusions}

The effectiveness of several active and passive devices to control flow in an adverse pressure gradient with secondary flows present was evaluated. In this study, passive micro vortex generators, micro bumps. and piczoelectric synthetic jets were evaluated for their flow control characteristics using surface static pressures. flow visualization. and 3D Stereo Digital Particle Image Velocimetry. Data also were acquired for synthetic jet actuators in a zero flow environment. The conclusions are summarized as follows:

1. The micro vortex generator is very effective in controlling the flow environment for an adverse pressure gradient. even in the presence of 
2t Greenblatt. D. and Wygnanski, I. "Parameters Affecting Dynamic Stall Control by Oscillatory Excitation," AIAA 99-3121. June. 1999.

25. Wygnanski, I, "Some New Observations Affecting the Control of Separation by Periodic Excitation." AIAA 2000-2314. June. 2000

20. Lin, J. C. "Control of Turbulent Boundary-Layer Separation Csing Micro Vortex Generators," AIAA $99-3404$. June. 1999

27 Joslin, R D, Horta, I, G, and Chen, F, J, "Transitioning Active Flow Control to Applications," AIAA 99-3575. June, 1909
28. Bryant, R G, Fox, R, 1.. Lachowicy, J $T$, and chen, F J., "Piezoelectric Synthetic Jets for Aircrati Control Surfaces," SPIE Proceedings. Vol 3674, 1909. PP 220-227

29. Instruments and Apparams. "Patt I - Measurement Uncertainty," ANSU/ASME PTC $191-1985$, American National Standards Inst. 1985

30. Lourenco, L. M., and Krothapalli, A. "True Resolution PIV A Mesh-Free Second Order Accurate Algorithm," Proceedings of the $10^{\text {th }}$ International Symposium on Application Techniques in Fluid Mechanics, Lisbon. July 2000

Table 1. Measurement Uncertainty

\begin{tabular}{|l|l|l|l|}
\hline Temperature, deg F & \pm 0.1 & $\mathrm{C}_{\mathrm{P}}$ & \pm 0.001 \\
\hline Density, slug/ft & \pm 0.00001 & PIV velocity components, $\mathrm{ft} / \mathrm{sec}$ & \\
\hline [otal pressure, $\mathrm{psi}$ & \pm 0.01 & Streamwise & \pm 5.2 \\
\hline Dynamic pressure, $\mathrm{psi}$ & \pm 0.01 & Vertical & \pm 2.6 \\
\hline Tunnel velocity, ft/sec & \pm 1.3 & Lateral & \pm 2.6 \\
\hline
\end{tabular}

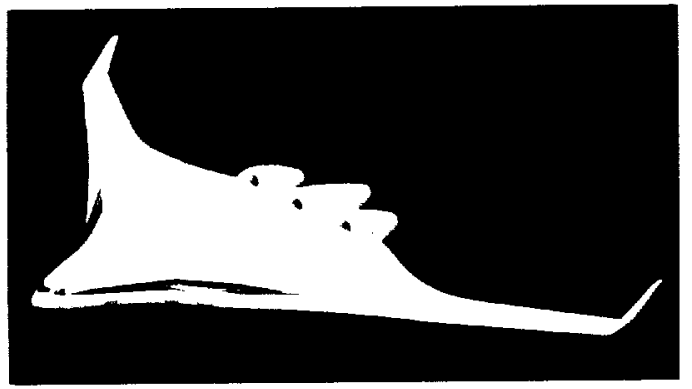

Figure 1. Blended Wing Body configuration.

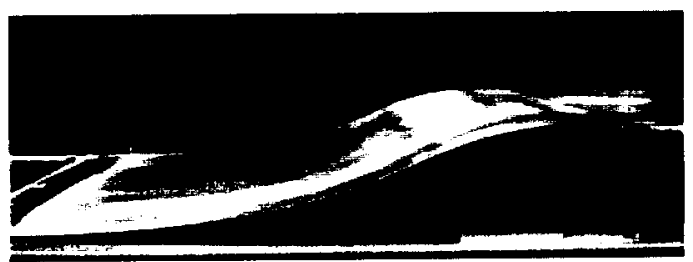

Figure 2 Adverse pressure gradient ramp installed in the 15 Inch Low Speed Tunnel

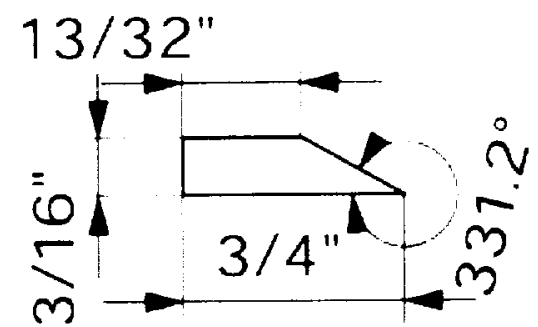

Figure; Micro vortex generator protile

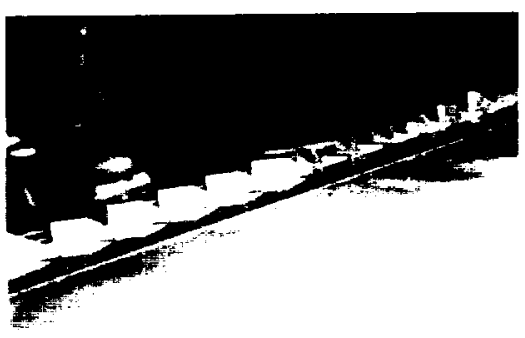

Figure 4 Micro vortex generator (MVG's) contiguration.

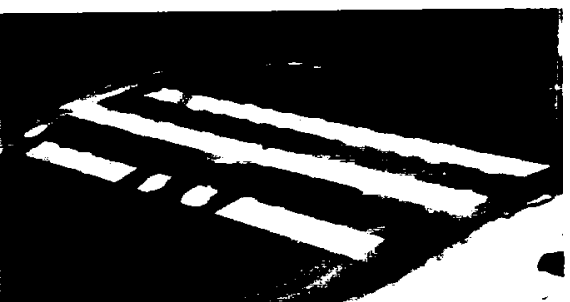

Figure 5. Micro bump configuration.

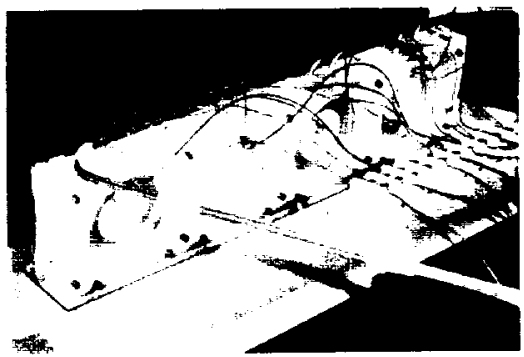

Figure 6 Piezoelectric synthetic jet configuration 

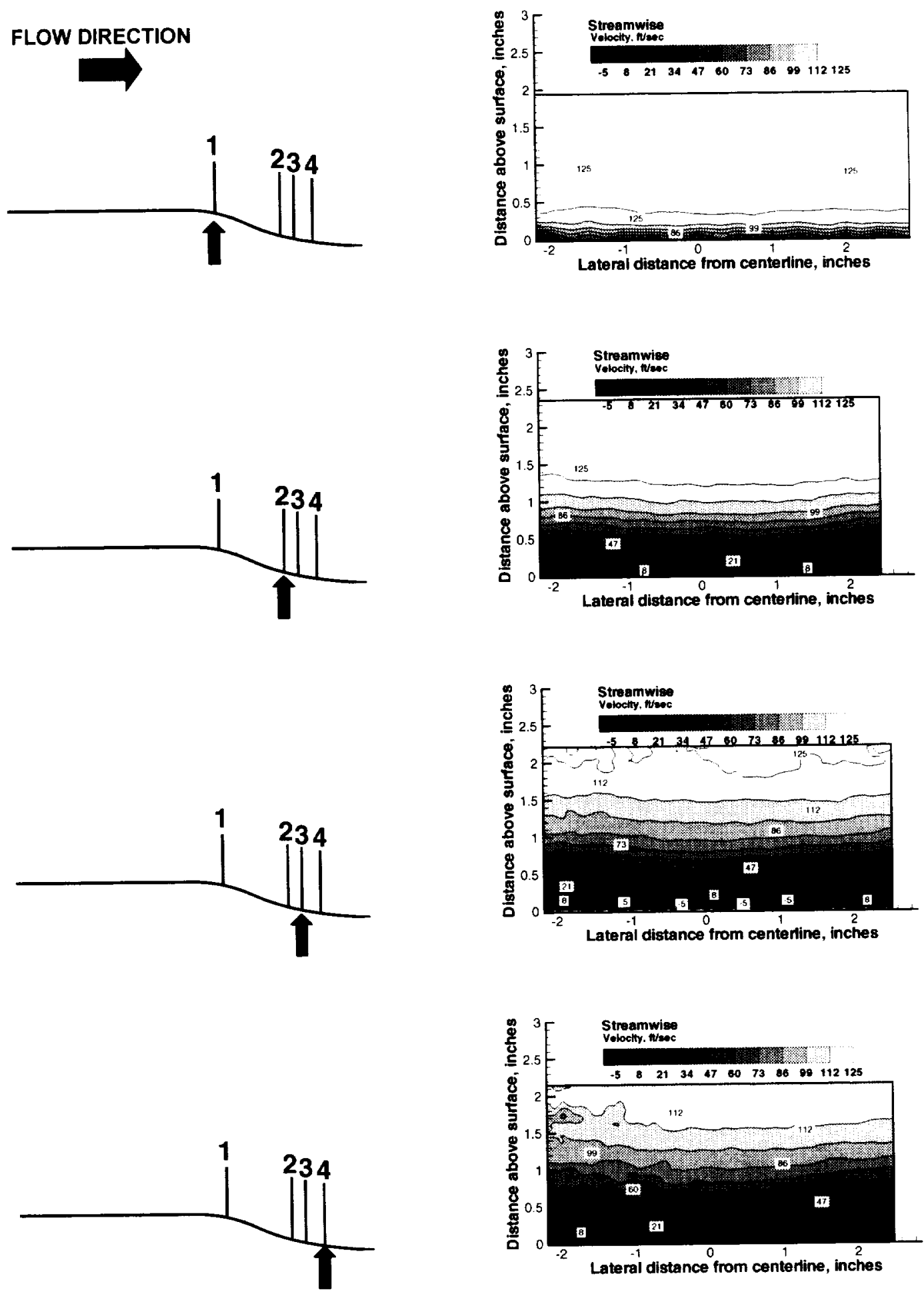

Figure 9. Velocity contours for baseline ramp at measurement stations $X=61.75 . X=66.90 . X=68.00$. and $X=69.50$. 
MVG's

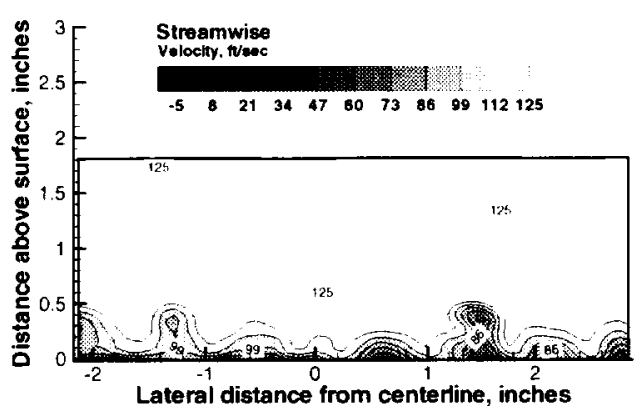

$$
X=61.75
$$
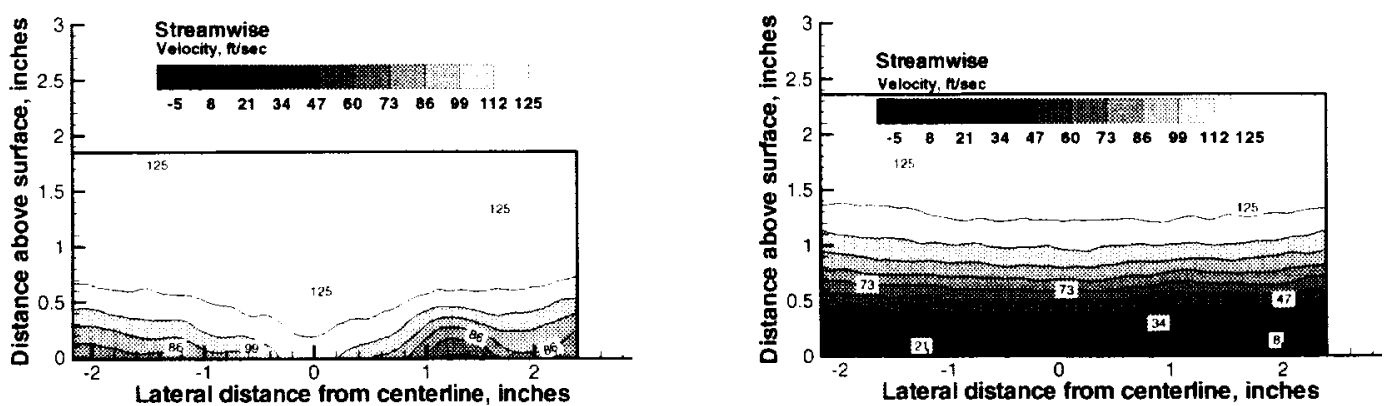

$X=66.90$
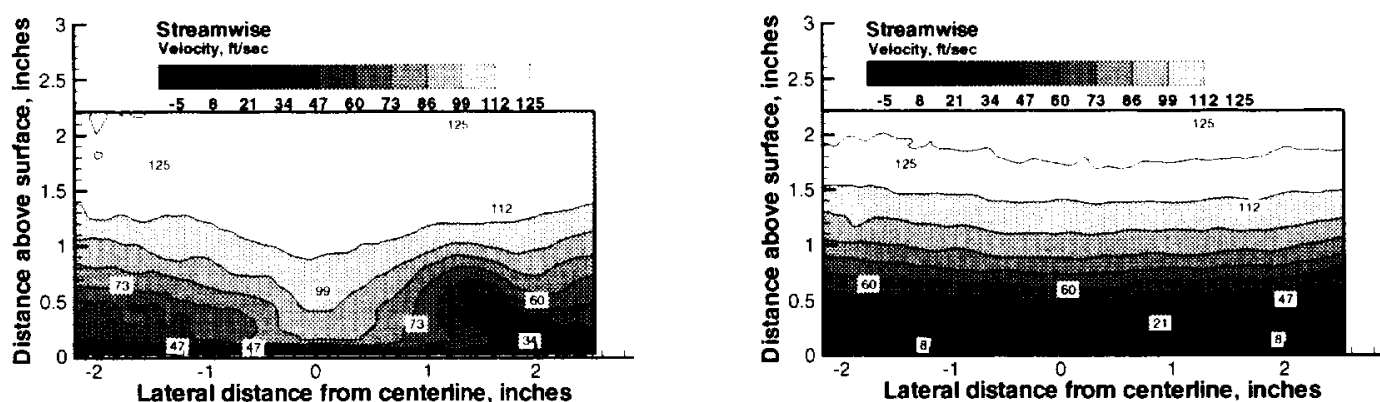

$X=68.00$
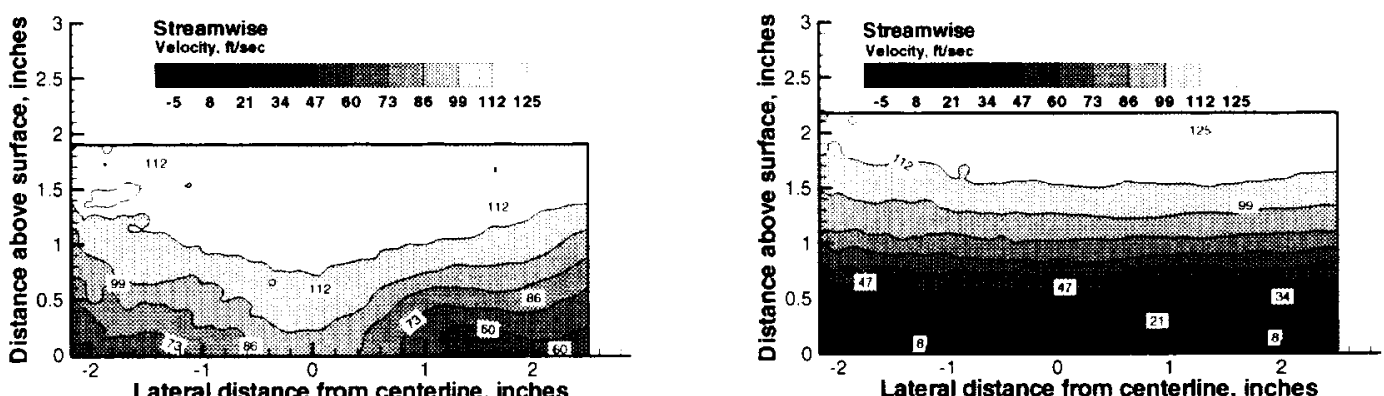

$X=69.50$

Figure 14. Comparison of streamwise velocity contours for MVG's and synthetic jets at stations $X=61.75 . X=66.90$. $X=68.00$. and $X=69.50$.

American Institute of Aeronautics and Astronautics 

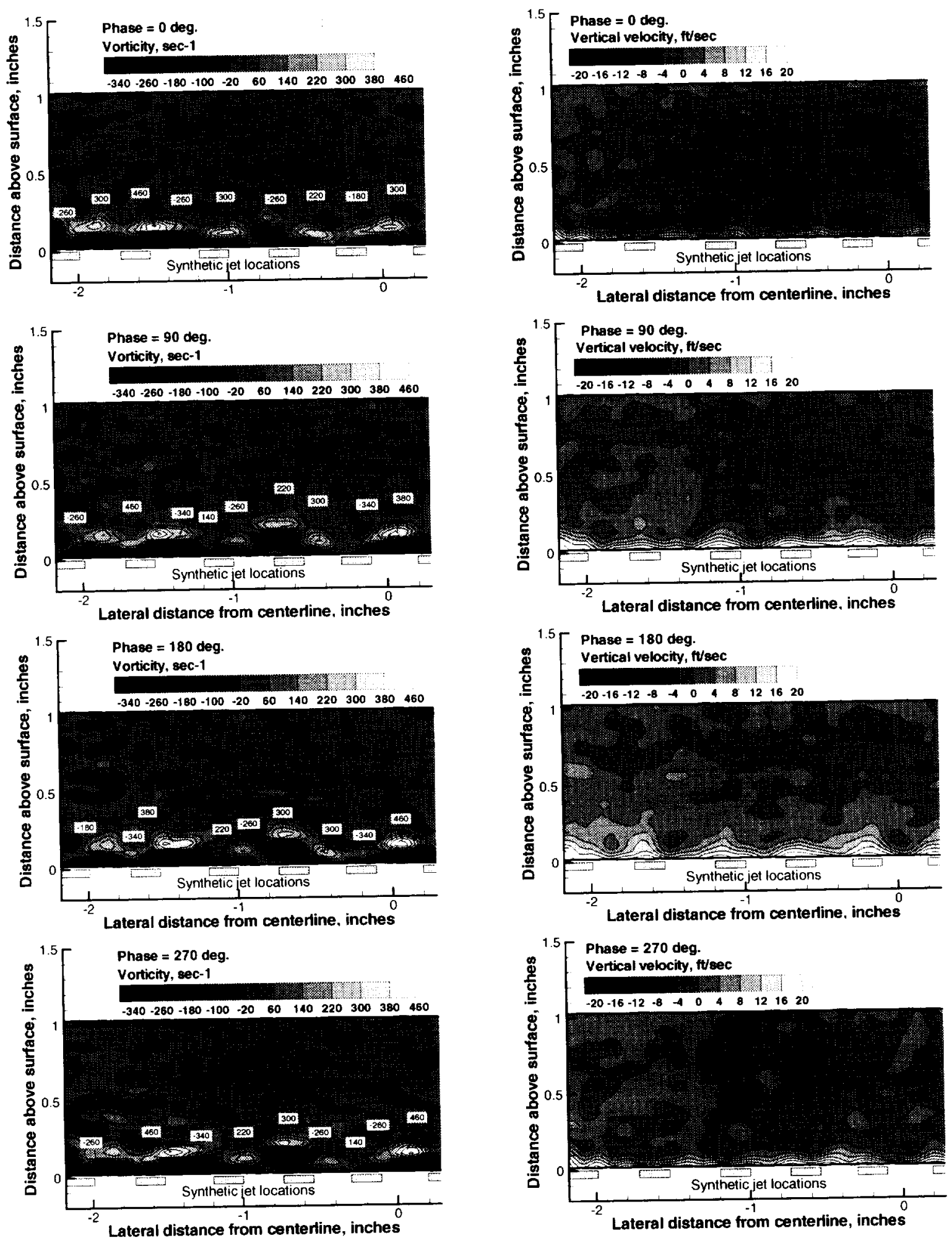

Figure 16. Phase-locked vorticity and vertical velocity contours of the synthetic jet actuators operating in a $140 \mathrm{ft} / \mathrm{sec}$ onset-flow. 
$700 \mathrm{~Hz}$
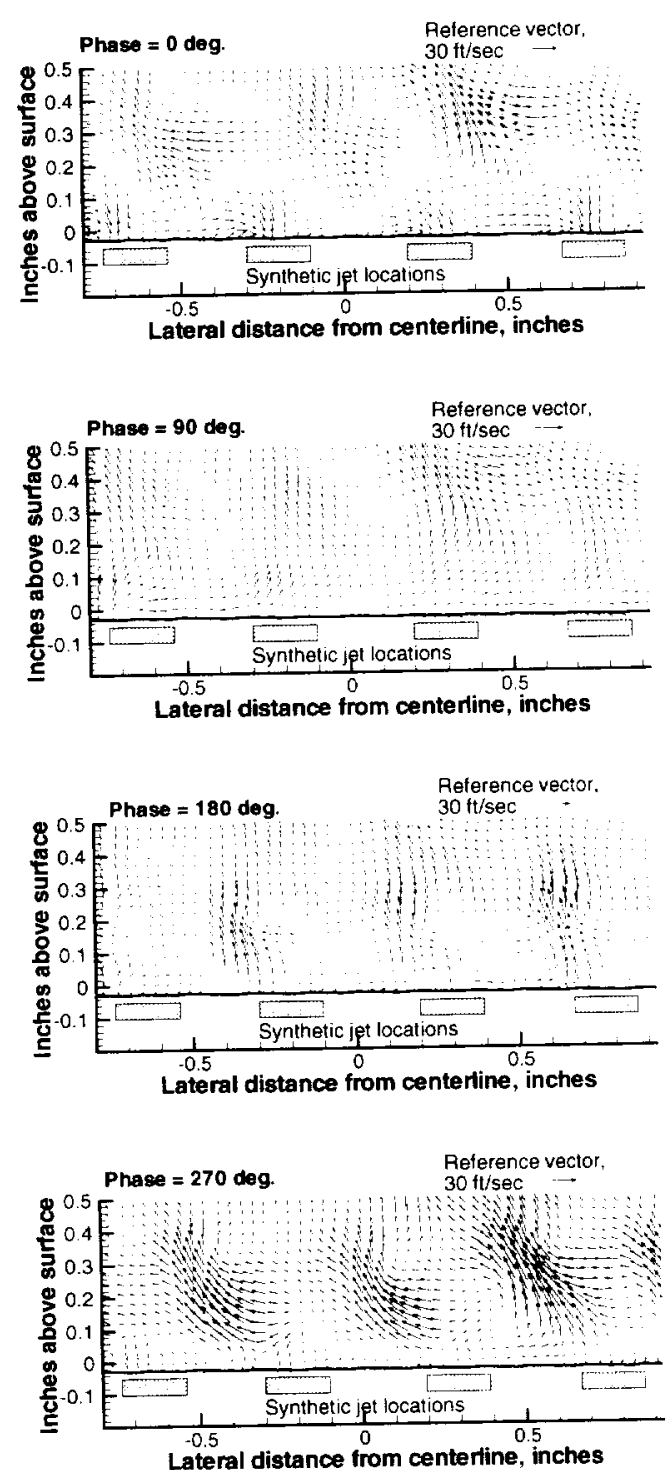

$300 \mathrm{~Hz}$
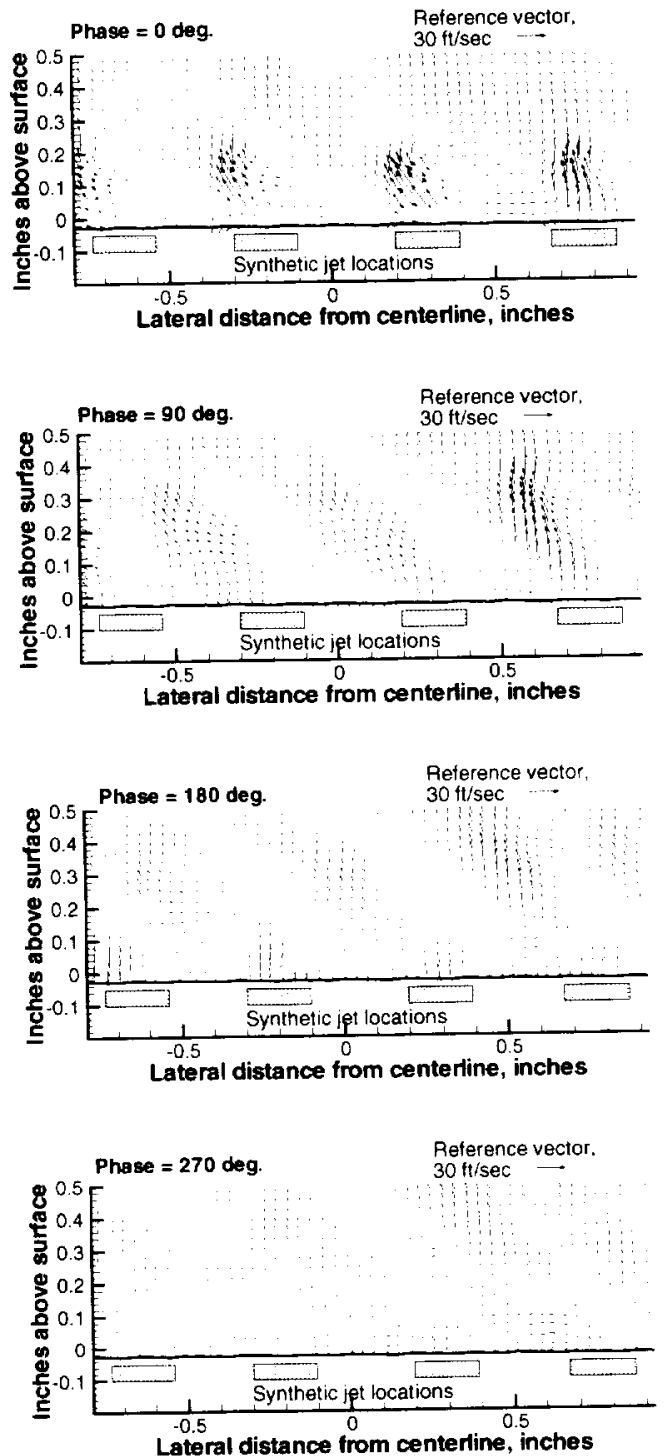

Figure 20. Phase-locked velocity vectors for synthetic jet actuators operating at $700 \mathrm{~Hz}$ and $300 \mathrm{~Hz}$ in zero onset flow. 


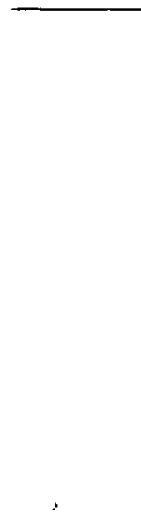

